\title{
Encapsulation and characterization of Garcinia atroviridis rinds water extracts loaded nanoparticles
}

\author{
${ }^{2}$ Yusof, N.B. and ${ }^{1,3, *}$ Abdul Aziz, A. \\ ${ }^{1}$ Institute of Bioproduct Development, Universiti Teknologi Malaysia, Skudai, Johor, Malaysia. \\ ${ }^{2}$ Schools of Chemical Engineering, Universiti Teknologi Malaysia, Skudai, Johor, Malaysia \\ ${ }^{3}$ Department of Chemical and Environmental Engineering, Malaysia-Japan International Institute of \\ Technology, Universiti Teknologi Malaysia, Kuala Lumpur, Malaysia
}

\begin{abstract}
Article history:
Received: 16 March 2020

Received in revised form: 29

May 2020

Accepted: 30 August 2020

Available Online: 5

September 2020

\section{Keywords:}

Garcinia atroviridis,

Hydroxycitric acid (HCA),

Chitosan nanoparticle,

Sodium tripolyphosphate

(TPP),

Characterizations.
\end{abstract}

DOI:

https://doi.org/10.26656/fr.2017.4(S2).S08

\begin{abstract}
Garcinia atroviridis fruit has been shown to express anti-obesity activity as a result of its bioactive compound, hydroxycitric acid (HCA). HCA is effective in decreasing appetite, inhibiting fat synthesis, and reducing body weight. However, HCA is very unstable towards certain conditions thus limiting its bioavailability. To overcome the issue of HCA instability, HCA was encapsulated in chitosan (CS) nanoparticles in this study. CS nanoparticles were prepared based on ionic gelation using sodium tripolyphosphate (TPP) as a cross-linking agent. The concentration of chitosan and TPP: chitosan volume ratios were varied and the resulting nanoparticles were characterized based on zeta potential, particle size, encapsulation efficiency (EE\%), and kinetics release. The most optimum nanoparticle was obtained with a combination of $1.5 \mathrm{mg} / \mathrm{mL}$ chitosan with a CS: TPP volume ratio of $4: 1$. Zeta potential was measured by approximately $49 \mathrm{mV}$. The size of the particle at optimum condition was found to be $140 \mathrm{~nm}$ and the nanoparticle had high encapsulation efficiency $(87.55 \pm 5.35 \%)$. G. atroviridis extract release from CS nanoparticles followed either Higuchi or Korsmeyer Peppas kinetic model. FT-IR studies indicated that $G$. atroviridis was encapsulated in CS nanoparticles. The present study revealed that concentration of chitosan, and CS: TPP volume ratio can significantly change the physical characteristics of the nanoparticles and this provides an avenue for formulators to engineer CS nanoparticles according to needs.
\end{abstract}

\section{Introduction}

Garcinia atroviridis (Gaertn.) Desr. (Clusiaceae) or its local name as Asam gelugor, is large polygamous trees or shrubs which can be found in tropical Asia, Africa, and Polynesia. For many years, the fruit rind is used traditionally as a food preservative, seasoning agent, or food-bulking agent, and also as a traditional remedy to treat constipation, piles, and rheumatism (Semwal et al., 2015). Currently, (-) - Hydroxycitric acid (abbreviated herein as HCA), an isolated derivative of these fruit rinds, has been incorporated into a wide range of pharmaceutical or nutraceutical product as a weight loss supplement. Studies have shown that the rind of Garcinia species has been found to contain approximately $10-30 \%$ of HCA (Jena et al., 2002). HCA is believed to have an anti-obesity activity that will result in reduced food intake, increased fat oxidation, and decreased de novo lipogenesis (Semwal et al., 2015).
Many types of research have investigated the physicochemical and biochemical effects of HCA, and from their studies, they believed that among other physiological effects, HCA could accelerate metabolism, improve glucose metabolism, and suppress appetite. A clinical study conducted by Roongpisuthipong et al. (2007) on 25 obese Thai women showed a decrease in triceps skinfold d, biceps skinfold, subscapular skinfold, upper arm circumference, percentage of body fat and body fat weight after consumption of HCA. According to Jena et al. (2002), the biological effect of HCA initiates the inhibition of adenosine triphosphate-citrate lyase (ATP), a catalyst for the extramitochondrial cleavage of citrate to oxaloacetate and acetyl-coenzyme A (acetylCoA). Acetyl-CoA is important in fatty acid, cholesterols, and triglyceride synthesis (Braswell and Atlanta, 1999). Thus, limiting the availability of acetylCoA units will suppress de novo fatty acid synthesis and lipogenesis. During metabolism, citrates are converts 
into acetyl coenzyme A with the help of an enzyme system, citrate lyase. When HCA is fed throughout this phase, part of the enzyme will be utilized by this acid, and thus the formation of acetyl coenzyme $\mathrm{A}$ is decelerated. Therefore, consumption of (-) hydroxycitric acid brings about a slowing of fat synthesis as well as fat accumulation.

In general, HCA takes two forms which are, free HCA, which is active but has a tendency to be unstable, and the more stable but inactive form of HCA-lactone. According to a study that was conducted by Moffet et al. (1997), the concentration of HCA from $G$. atroviridis is $23-54 \%$, with $6-20 \%$ of HCA-lactone. The free HCA tends lactonization during drying, concentration, and evaporation. However, to be effective, hydroxycitric acid must be in straight-chain form and not as a lactone (Verhoeckx et al., 2015). According to Gokaraju et al. (2005), the lactone form of HCA does not possess the desired bioactivity for weight loss purposes since the lactone form lacks the appropriate affinity for the receptor which is the target of the actions of HCA.

To overcome the problems associated with the stability of HCA, encapsulating HCA in a nano-carrier can be considered. Nano-encapsulation technology has the potential for improve the delivery of bioactive compounds. Nano-particulate systems possess the potential to enhance the bioavailability, increase the therapeutic effect of the substances, controlled release, site-specific bioactive compound delivery, and sustained release of the substances over a longer period (Hamidi et al., 2008). Since nanoparticles have a larger surface to volume ratio compared to the bulk material, the dose and frequency of administration would be reduced, hence increasing patient compliance (Xianyi et al., 2012).

Chitosan is a biopolymer that is often employed in the production of nanoparticles owing to their biocompatibility and biodegradability. It is a linear cationic polysaccharide consisting of $(1 \rightarrow 4)$ linked units of glucosamine and $\mathrm{N}$-acetyl-glucosamine, which is mainly produced through partial deacylation of chitin. According to Mislick and Baldeschwieler (1996), the cationic polymer can be used in both membrane adhesion and lysosomal escape of the encapsulated DNA.

Chitosan nanoparticles are attractive as potential drug carriers due to their positive attributes, such as biocompatibility, mucoadhesiveness, and mild preparation process (Sawtarie et al., 2017). These nanoparticles can also encapsulate and protect sensitive payloads from denaturation, thus preserving the bioactivity of their compounds. Chitosan is a naturally occurring polysaccharide that is commonly derived from crustacean shells from prawns or crabs. This biocompatible and biodegradable cationic polymer has been approved by the U.S. FDA for tissue engineering and drug delivery purposes (Mohammed et al., 2017). The positive charge from the amine group of chitosan makes it possible to form nanoparticles by ionotropic gelation with polyanion such as sodium tripolyphosphate (TPP) (Jonassen 2012). Appropriate sized chitosan nanoparticles can penetrate deep into tissues through fine capillaries and cross the fenestration in the epithelial lining of the liver (Vinagradov et al., 2002). TPP was also considered as nutritious since the polyphosphates of TPP could hydrolyze into simpler phosphates (Kulakovskaya et al., 2012). Moreover, TPP also has been described to be neither mutagenic nor carcinogenic (Nagpal et al., 2013).

By mixing the diluent of chitosan and sodium tripolyphosphate (TPP), nanoparticles that range between tens to hundreds of nm can be generated. Chitosan/TPP nanoparticles are attractive as potential carriers for the drug delivery system due to their mucoadhesiveness properties and mild preparation method. In nanomedicine, particle properties such as the size and the surface charge play an important role in determining its biological behaviour. These properties affect cellular uptake, protein adsorption, and accumulation of the nanoparticles and their distribution throughout the body. However, chitosan-TPP nanoparticles can be polydispersed and suffer from poor stability, which limits their use (Nemrawi et al., 2018). Since efficient delivery of nanoparticles depends on both size and density of surface charge, there has been significant interest in controlling both their average size and surface charge (Quan et al., 2005). The particle size of chitosan depends on two crucial factors such as chitosan concentration and CS: TPP volume ratios.

The main purpose of this present study was to encapsulate the $G$. atroviridis rinds and to analyze the characteristics of the G. atroviridis rinds loaded chitosan nanoparticles. The effects of chitosan concentrations and CS: TPP volume ratios on the physical characteristics of CS were studied. Kinetic releases of the CS nanoparticles were studied as well.

\section{Materials and methods}

\subsection{Materials}

Dried G. atroviridis rinds were obtained from the Institute of Bioscience UPM. Chitosan low molecular weight deacetylated chitin, poly (D-glucosamine), Sodium tripolyphosphate (TPP) technical grade $85 \%$, Potassium hydroxycitrate tribasic monohydrate $\geq 95.0 \%$ standard, Dowex ${ }^{\circledR} 50 \mathrm{WX} 8$ hydrogen form, $200-400$ mesh, Dialysis sacks (average flat width $25 \mathrm{~mm}$ (1.0 in.) 
MWCO 12,000 Da) was purchased from Sigma Aldrich (Germany). All chemicals were used as received unless otherwise noted.

\subsection{Preparation of CS loaded Garcinia atroviridis nanoparticle suspension}

The nanoparticles were prepared based on ionic gelation of chitosan with sodium tripolyphosphate (TPP) anions as described by (Jonassen et al., 2012) with slight modifications. Chitosan was dissolved in an aqueous solution of $1 \%$ acetic acid in different concentrations to form $0.5 \mathrm{mg} / \mathrm{mL}, 1.5 \mathrm{mg} / \mathrm{mL}$, and $3 \mathrm{mg} / \mathrm{mL}$ of chitosan solution as shown in Table 1. In another beaker, TPP at a constant concentration $(0.7 \mathrm{mg} / \mathrm{mL})$ was dissolved in deionized water together with $0.2 \% \mathrm{w} / \mathrm{v}$ of the active ingredient, G. atroviridis. Chitosan nanoparticles were spontaneously obtained when the TPP solution was added dropwise into the chitosan solution under magnetic stirring for 1 hour at room temperature. Chitosan: TPP volume ratios were varied as either $4: 1$ or 7: 1 . The solution was then sonicated at $10 \%$ amplitude for 3 mins using a probe-type (Model 500 Digital Sonic Dismembrator $117 \mathrm{~V} / 60 \mathrm{~Hz}, 4.0$ A, Fischer Scientific, UK).

\subsection{Nanoparticles characterization}

\subsubsection{Particle size, PDI and zeta potential}

The mean particle size (PS), polydispersity index (PDI), and zeta potential (ZP) of the nanoparticles were measured using a particle sizer (Zetasizer Nano ZS Malvern, UK) based on dynamic light scattering (DLS). The parameters for measurement and calculation were set as follows: $173^{\circ}$ backscatter measurement angle, 1.33 material refraction index, $25^{\circ} \mathrm{C}$, and water as the dispersion medium. Each sample was measured 3 times in a clear disposable cell. The number of runs and duration were optimized for each sample to obtain results that meet the measurement quality criteria. The samples were dissolved in deionized water with (1/10 dilution) before measurement (Ling et al., 2012).

\subsubsection{Determination encapsulation efficiency Garcinia atroviridis in CS nanoparticles}

The concentration of HCA inside the G. atroviridis was determined using HPLC. Potassium hydroxycitrate tribasic monohydrate was used as a reference standard to detect the percentage of HCA compounds in $G$. atroviridis rinds extract. The standard was suspended in $10 \mathrm{~mL}$ of deionized water and was treated with $25 \% \mathrm{w} / \mathrm{v}$ of Dowex $50[\mathrm{H}+]$ (Jayaprakasha et al., 2003). The mixture was centrifuged at $1000 \mathrm{rpm}$ for 5 mins. The supernatant was collected, and the resin was washed to reach neutral $\mathrm{pH}$. Washing and supernatant were combined, made up to a volume of $200 \mathrm{~mL}$, filtered and stored at $4^{\circ} \mathrm{C}$ for further use. The preparation of standard followed the method by Jayaprakasha and Sakariah (2000) with slight modifications. Samples or standards were filtered using $0.45 \mu \mathrm{m}$ filters before analysis. Standard solutions or samples $(20 \mu \mathrm{L})$ were injected in an HPLC instrument (Shimadzu, Japan) that employed a reverse - phase $\mathrm{C}_{18}$ column ( $4.6 \times 250 \mathrm{~mm}$, Waters) and $6 \mathrm{mM}$ sulphuric acid as the mobile phase at a flow rate of $1.0 \mathrm{~mL} / \mathrm{min}$ with $\mathrm{UV}$ detection at $210 \mathrm{~nm}$.

To determine the encapsulation efficiency of HCA, the encapsulated CS-TPP was separated from nonencapsulated HCA using the size exclusion spin minicolumn method. CS nanoparticles were separated from non-encapsulated HCA using a spin minicolumn packed with Sephadex G-50 (Epoch Life Science, USA). Before the minicolumn was ready to be used, the spin minicolumn was centrifuged first at $2000 \mathrm{rpm}$ for 2 mins to eliminate excess water. Then, $0.1 \mathrm{~mL}$ solution of CS nanoparticles were loaded into the minicolumn and the centrifugation was repeated. The expelled nanoparticles were then treated with methanol and their HCA content measured using HPLC. Encapsulation efficiency is calculated as the ratio of HCA content within CS to the total HCA content in the suspension Equation (1):

Encapsulation Efficiency $(\%)=\frac{(\text { The total amount of G.atroviridis) }- \text { (free G.atroviridis) }}{\text { Total amount G.atroviridis }} \times 100$

\subsection{Morphology-TEM analysis}

The morphology of CS nanoparticles was viewed using Transmission Electron Microscopy (TEM) at $100 \mathrm{kV}$ accelerating voltage. A droplet of CS suspension was placed onto a copper grid and was allowed to absorb for 3 mins. The remaining sample on the copper grid was bloated with a filter paper and dried in a desiccator for 1 hr.

\subsection{Fourier Transform - infrared (FTIR) spectroscopy}

FTIR analysis was conducted for pure chitosan, TPP, unloaded CS nanoparticles, and G. atroviridis loaded CS nanoparticles. Transmittance spectra were obtained using (Shimadzu IRT racer-100) fitted with attenuated total reflectance mode (ATR) cell. The IR absorbency scans were examined between 700 and $4000 \mathrm{~cm}^{-1}$ (Fabio and Marta, 2003).

\subsection{Kinetic release of HCA in Chitosan-TPP nanoparticle}

In vitro release of HCA from CS-TPP suspensions was examined separately using the dialysis bag technique. Dialysis bags with $12 \mathrm{kDa}$ MWCO have been used for this analysis. The procedures to evaluate its kinetics release were followed by a method proposed by 
(Fathi et al., 2013; Ezhilarasi et al., 2016) with slight modifications. The PBS with $100 \mathrm{mM}$, at $\mathrm{pH}$ of 7.4 was used as a release medium. The dialysis bags were soaked in distilled water for $12 \mathrm{hrs}$ before further use. $5 \mathrm{~mL}$ of CS-TPP loaded HCA and unencapsulated HCA were sealed in the dialysis bag and immersed in $20 \mathrm{~mL}$ of PBS release medium. The samples were placed in a thermostatic shaker at $37^{\circ} \mathrm{C}$ and $100 \mathrm{rpm}$. An aliquot of 1 $\mathrm{mL}$ of release medium was withdrawn at time intervals such as $0,0.5,1,2,3,4,5,6,7,8,910,11,12,24 \mathrm{hrs}$ and the same amount of PBS medium is replaced immediately to maintain the sink conditions. The HCA release at various time intervals was evaluated using the HPLC method. The cumulative percentage of HCA was determined using the following equation:

Cumulative relative $(\%)=\frac{\text { cumulative amount of } H C A}{\text { initial amount of } H C A} \times 100$

To understand the release kinetic of HCA from CSTPP nanoparticles, the release data were fitted to the following kinetics model:

$$
\begin{array}{ll}
\text { Zero }- \text { order } & : \mathrm{C}=k_{\mathrm{o}} \mathrm{t} \\
\text { First }- \text { order } & : \log \mathrm{C}=\log \mathrm{C}_{\mathrm{o}}-\mathrm{Kt} / 2.303 \\
\text { Higuchi } & : \mathrm{Q}=\mathrm{Kt}^{1 / 2} \\
\text { Peppas -Korsmeyer } & : \mathrm{M}_{\mathrm{t}} / \mathrm{M} \infty=\mathrm{Kt}^{\mathrm{n}} \\
\text { Hixson-Crowell } & : \sqrt[3]{\mathrm{W}_{\mathrm{o}}-}{ }^{3} \sqrt{\mathrm{W}_{\mathrm{t}}}=\mathrm{K}_{\mathrm{s}} \mathrm{t}
\end{array}
$$

\subsection{Statistical analysis}

The measurements were repeated at least three times and data were expressed as mean $\pm \mathrm{SD}$. Data were analyzed using one-way analysis of variance (ANOVA), and value of $\mathrm{P}<0.05$ was considered as statistically significant.

\section{Results and discussion}

3.1 The effects of formulation variables on the physical characteristics of CS-nanoparticles

In this present work, a series of experiments were conducted to study the characteristics of the nanoparticles based on particle size, PDI, surface charge (zeta potential), encapsulation efficiency percentage $(\mathrm{EE} \%)$, and kinetic release profile. Different concentrations of chitosan were varied at $0.5 \mathrm{mg} / \mathrm{mL}, 1.5$ $\mathrm{mg} / \mathrm{mL}$, and $3.0 \mathrm{mg} / \mathrm{mL}$, while a constant concentration of TPP was used $(0.7 \mathrm{mg} / \mathrm{mL})$. TPP's concentration was chosen based on the highest value appropriate for crosslinking as suggested by Purwantiningsih et al. (2015). Chitosan nanoparticles were spontaneously obtained when the TPP solution was added dropwise into the chitosan solution under magnetic stirring for $1 \mathrm{hr}$ at room temperature at the selected chitosan to TPP ratios of $4: 1$ and $7: 1$. The results of particle size and PDI are shown in Table 1.

The size of a nanoparticle is a crucial characteristic that needs to be studied since it affects the drug circulation in the blood, penetration across physiological drug barriers, site and cell-specific localization, and induction of cellular response (Wang et al., 2011). In general, the size of a non-spherical nanomaterial is defined as an equivalent diameter of a spherical particle whose selected physical properties are equivalent to those of nanomaterial in the same environment (Powers et al., 2006). The results indicated that formulation variables (CS concentration and CS: TPP volume ratio) influence particle size distributions (Table 1). The particle sizes obtained ranged from $140 \mathrm{~nm}$ to $380 \mathrm{~nm}$. The nanoparticles (CS-TPP 0.5, 4:1 and CS-TPP 0.5, $7: 1)$ which were prepared at the lowest concentration of chitosan and the volume ratios of either $4: 1$ or $7: 1$ were found to have a particle size of $196 \mathrm{~nm}$ and 182, respectively. When the concentration of chitosan was increased to $1.5 \mathrm{mg} / \mathrm{mL}$ and using a volume ratio of $4: 1$, smaller nanoparticles $(140 \mathrm{~nm})$ with a low polydispersity index $(0.28)$ were formed, indicating that the particle size distributions were narrower. However, when the volume ratio of CS: TPP was changed to $7: 1$ at $1.5 \mathrm{mg} / \mathrm{mL}$ chitosan concentration, the particle size and PDI increased to $194 \mathrm{~nm}$ and 0.43, respectively. Further increase in the concentration of chitosan to $3.0 \mathrm{mg} / \mathrm{mL}$, resulted in a further increase in the particle size of the CS nanoparticles. Out of all formulations, nanoparticles obtained using $1.5 \mathrm{mg} / \mathrm{mL}$ of chitosan at the ratio of CS: TPP of 4:1 showed the smallest particle size and distribution.

A possible explanation for these results is that the

\begin{tabular}{|c|c|c|c|c|c|c|}
\hline ID & Chitosan $(\mathrm{mg} / \mathrm{mL})$ & $\mathrm{TPP}(\mathrm{mg} / \mathrm{mL})$ & CS:TPP ratio & $\begin{array}{l}\text { Garcinia atroviridis } \\
(\mathrm{mg} / \mathrm{mL})\end{array}$ & Particle size (nm) & PDI \\
\hline CS-TPP 0.5/4:1 & 0.5 & 0.7 & $4: 01$ & 2 & $196.13 \pm 0.7$ & $0.30 \pm 0.03$ \\
\hline CS-TPP 0.5/7:1 & 0.5 & 0.7 & $7: 01$ & 2 & $181.55 \pm 5.5$ & $0.43 \pm 0.01$ \\
\hline CS-TPP 1.5/4:1 & 1.5 & 0.7 & $4: 01$ & 2 & $140.35 \pm 0.2$ & $0.28 \pm 0.0$ \\
\hline CS-TPP 1.5/7:1 & 1.5 & 0.7 & $7: 01$ & 2 & $194.25 \pm 2.6$ & $0.43 \pm 0.01$ \\
\hline CS-TPP 3.0/4:1 & 3 & 0.7 & $4: 01$ & 2 & $350.10 \pm 2.3$ & $0.35 \pm 0.01$ \\
\hline CS-TPP 3.0/7:1 & 3 & 0.7 & 7:01 & 2 & $376.00 \pm 4.5$ & $0.45 \pm 0.02$ \\
\hline
\end{tabular}
numbers of the amine group from the chitosan was enough for cross-linking with the polyanion from the TPP, which caused a greater electrostatic interaction,

Table 1. CS- TPP formulations, average particle size (nm), and polydispersity index (PDI). 
thus resulting in narrow size distributions (Masaruddin et al., 2015). Whereas at a lower concentration of chitosan, the cationic amine groups of chitosan were limited for crosslinking with the polyanion of TPP. This led to low electrostatic interaction, hence resulting in a larger size with broad size distribution. At a high concentration of chitosan (exceed than $3.0 \mathrm{mg} / \mathrm{mL}$ ), amine groups of chitosan exceeded the TPP, so it limited the amount of polyanion available for cross-linking, thus leading to the formation of a larger particle with a high polydispersity (Bhumkar and Pokharkar, 2006; Jonassen et al., 2012).

Aside from the particle size distribution, the surface charge is also a factor that affects the nanoparticle's interaction with biological systems including cellular uptake (Bhattacharjee et al., 2016). Zeta potential (ZP) measurement is a good way to evaluate electrostatic stabilization of suspensions where it measured the charge on a particle at the shear plane. ZP value of at least \pm 30 $\mathrm{mV}$ indicates the stability of the nanoparticle suspension (Kaszuba et al., 2010). Based on the obtained results, zeta potential for all formulations was positive, ranging from $+24.25 \mathrm{mV}$ to $+49.57 \mathrm{mV}$. The highest zeta potential was detected upon the combination of chitosan with a concentration of $1.5 \mathrm{mg} / \mathrm{mL}$ and the ratio of $4: 1$ of CS: TPP. The zeta potential for CS-TPP 1.5/4:1 was + $49.57 \mathrm{mV}$ which indicated high stability. While the lowest zeta potential was obtained when the nanoparticles (CS- TPP 0.5/7:1) was formulated using the lowest concentration of chitosan in this study. The results are shown in Figure 1. The high zeta potential might be because when chitosan molecules were fully cross-linked, the particle became highly positively charged and this resulted in a very strong electrostatic

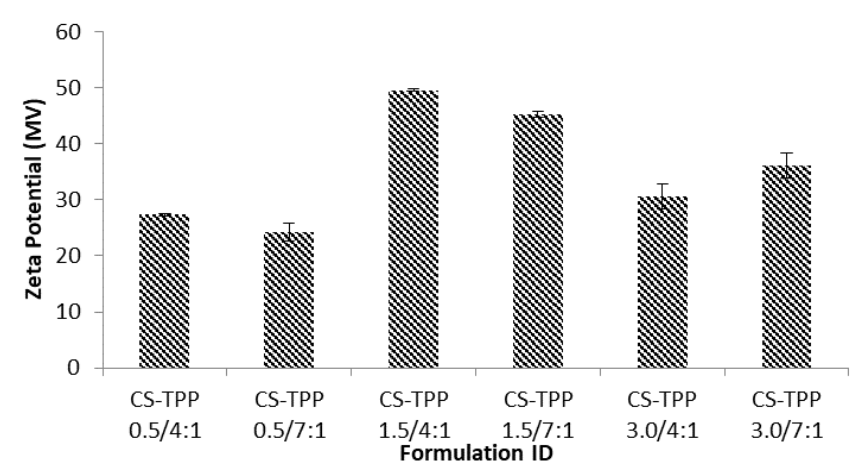

Figure 1. The surface charge (Zeta Potential) Measurements for the series of formulations. repulsion between particles with the same charge causing segregation of the particles.

Encapsulation efficiency is one of the most crucial parameters for nanoparticle study. In this analysis, the active compound of G. atroviridis which is encapsulated HCA was determined. Nanoparticles obtained using 1.5 $\mathrm{mg} / \mathrm{mL}$ of chitosan and 4:1 volume ratio of CS-TPP were the most efficient in encapsulating $G$. atroviridis extract compared to other formulations (Figure 2). The encapsulation efficiency of CS-TPP 1.5/4:1 and CS-TPP $1.5 / 7: 1$ was recorded at $87.6 \%$ and $86.4 \%$ respectively.

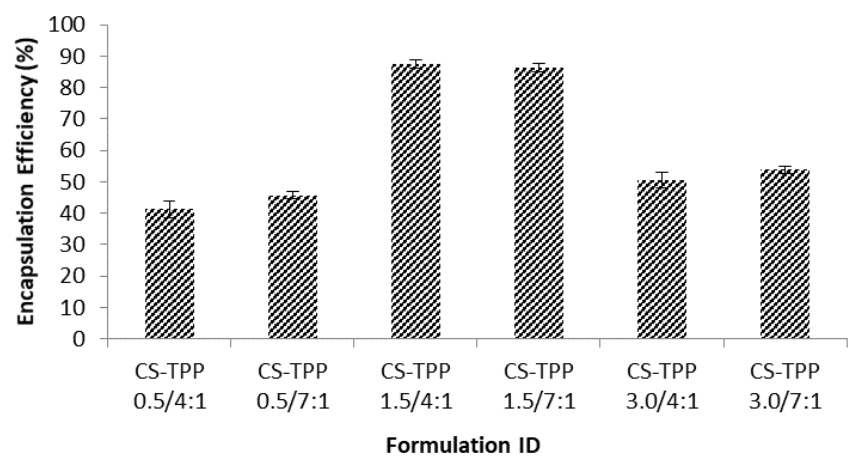

Figure 2. The encapsulation efficiency (EE\%) for the series of CS- nanoparticles formulations

The release profile of G. atroviridis rinds extract is shown in Figure 3. To predict the kinetics of $G$. atroviridis extract release from the CS- nanoparticles, various kinetic models were studied. The best release model was chosen based on the highest correlation coefficient $\left(\mathrm{R}^{2}\right)$ (Table 2). Based on the $\mathrm{R}^{2}$ values, the release of $G$. atroviridis rinds extract from CS-TPP 0.5/4:1, CS-TPP 0.5/7:1, CS-TPP 3.0/4:1, and CS-TPP 3.0/7:1 followed the Higuchi model. The nanoparticles obtained from the lowest concentration of chitosan (CSTPP 0.5/4:1 and CS-TPP 0.5/7:1) showed a burst release of $25 \%$ of the $G$. atroviridis extract in the $2^{\text {nd }} \mathrm{hr}$. This might be due to the accelerated release of unencapsulated G. atroviridis extract from the surface of a particle or in the suspension. Both nanoparticles showed continuous release of G. atroviridis extract up to $70.6 \%$ and $85.4 \%$ for CS-TPP 0.5/4:1 and CS:TPP 0.5/7:1 respectively. Higuchi model describes the release from the insoluble matrix as per square root of time-dependent process based on Fickian diffusion and this is a suitable mechanism for therapeutic agent release (Lokhandwala

Table 2. Evaluation of G. atroviridis water extract released from chitosan nanoparticles using structured models

\begin{tabular}{clccccc}
\hline Formulas & \multicolumn{7}{c}{ Zero order } & First order & Higuchi & Korsmeyer -peppas model & Hixson \\
\hline CD-TPP 0.5/4:1 & $\mathrm{R}^{2}$ & 0.75 & 0.78 & 0.87 & 0.83 & 0.77 \\
CS-TPP 0.5/7:1 & $\mathrm{R}^{2}$ & 0.82 & 0.89 & 0.92 & 0.83 & 0.87 \\
CS-TPP 1.5/4:1 & $\mathrm{R}^{2}$ & 0.91 & 0.94 & 0.91 & 0.96 & 0.93 \\
CS-TPP 1.5/7:1 & $\mathrm{R}^{2}$ & 0.84 & 0.86 & 0.86 & 0.95 & 0.85 \\
CS-TPP 3.0/4:1 & $\mathrm{R}^{2}$ & 0.82 & 0.87 & 0.92 & 0.89 & 0.85 \\
CS-TPP 3.0/7:1 & $\mathrm{R}^{2}$ & 0.83 & 0.88 & 0.93 & 0.88 & 0.87 \\
\hline
\end{tabular}


et al., 2013; Suvakanta et al., 2010).

On the other hand, the release from CS-TPP 1.5/4:1, and CS-TPP 1.5/7:1 followed the Korsmeyer-Peppas model. For the CS-TPP $1.5 / 4: 1$, the release reached a plateau by the $12^{\text {th }} \mathrm{hr}$ with $47.1 \%$ of G. atroviridis extract released. This formulation showed the slowest and most sustained release compared to other formulations. This might be due to the slow diffusion of $G$. atroviridis extract from the CS-TPP nanoparticle. Values of $n$ of $0.45<0.89$ for both formulations indicated that the release of $G$. atroviridis extract from CS-TPP 1.5/4:1, and CS-TPP 1.5/7:1 were non-fickian in nature.

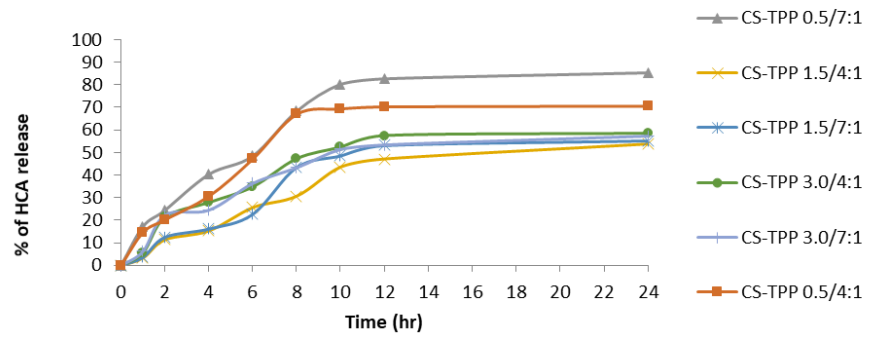

Figure 3. In-vitro release profile of HCA from CS-TPP nanoparticles in sink condition for $24 \mathrm{hrs}$ (sink condition, PBS, pH 7.4)

3.2 Morphological characteristics of chitosannanoparticles using Transmission Electron Microscopy (TEM)

Bioactive loading, release properties, and cellular uptake of a nanocarrier depend not only on the size and chemical composition but also on particle morphology. To confirm the spherical morphology of the prepared nanoparticles, images of the nanoparticles were taken using a Transmission Electron Microscope. According to Rampino et al. (2013), analyses using TEM provide the size of almost dehydrated particles, while measurements of dynamic light scattering provide an estimate of the particle size in a suspension. Figure 4 shows TEM micrographs of particles produced at different concentrations of chitosan $(0.5 \mathrm{mg} / \mathrm{mL}, 1.5 \mathrm{mg} / \mathrm{mL}, 3.0$ $\mathrm{mg} / \mathrm{mL}$ ) with $4: 1$, CS: TPP volume ratio. The images show that the particle size obtained correlate well with the particle size measured by dynamic light scattering using Zetasizer Nano ZS. For the CS-TPP 0.5/4:1, the image shows an aggregation of two particles. One of the reasons for this might be due to the low zeta potential of those particles which makes them stick with each other. While, for the CS-TPP 1.5/4:1 formulation, a particle with smooth spherical shape was observed. The obtained size was very similar to the size of the particle measured using DLS.

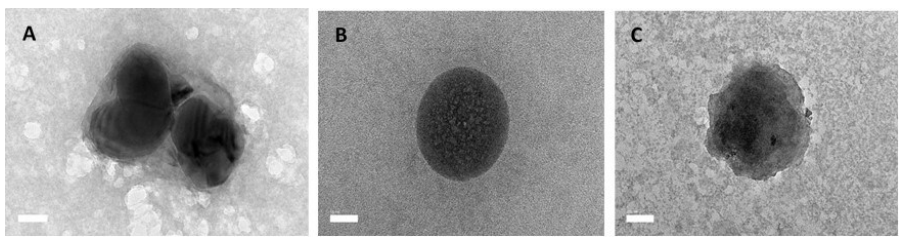

Figure 4. TEM micrograph of CS nanoparticles prepared from different CS concentration and CS: TPP volume ratio; a) CS:TPP 0.5/4:1; b) CS:TPP 1.5/4:1; c) CS:TPP 3.0/4:1

\subsection{Evaluation of the structure of components by Fourier Transform Infra-Red (FTIR)}

FT-IR analysis was done on TPP powder, CS powder, G. atroviridis water extract, and $G$. atroviridis water extract loaded CS. The results are shown in Figure 5. For TPP, the absorption bands at 1174.65 to 1091.71 $\mathrm{cm}^{-1}$ represent the $\mathrm{P}=\mathrm{O}$ groups and the band at 877.61 $\mathrm{cm}^{-1}$ indicates the P-O group. The FTIR spectrum of chitosan showed bands at 3309.85, 2825.72, 1624.06, $1332.89,1097,1013$, and $979.84 \mathrm{~cm}^{-1}$. The intense broad peak at $3309.85 \mathrm{~cm}^{-1}$ was the characteristic of hydrogenbonded $\mathrm{O}-\mathrm{H}$ stretch band and $\mathrm{NH}_{2}$ group. The broad peak was characterized by the hydroxyl functional group in alcohol and phenol compounds. This indicates that chitosan is surrounded by some proteins and secondary metabolites such as lignin sterols and alkaloids which have a functional group of hydroxyl, amines, alcohols,

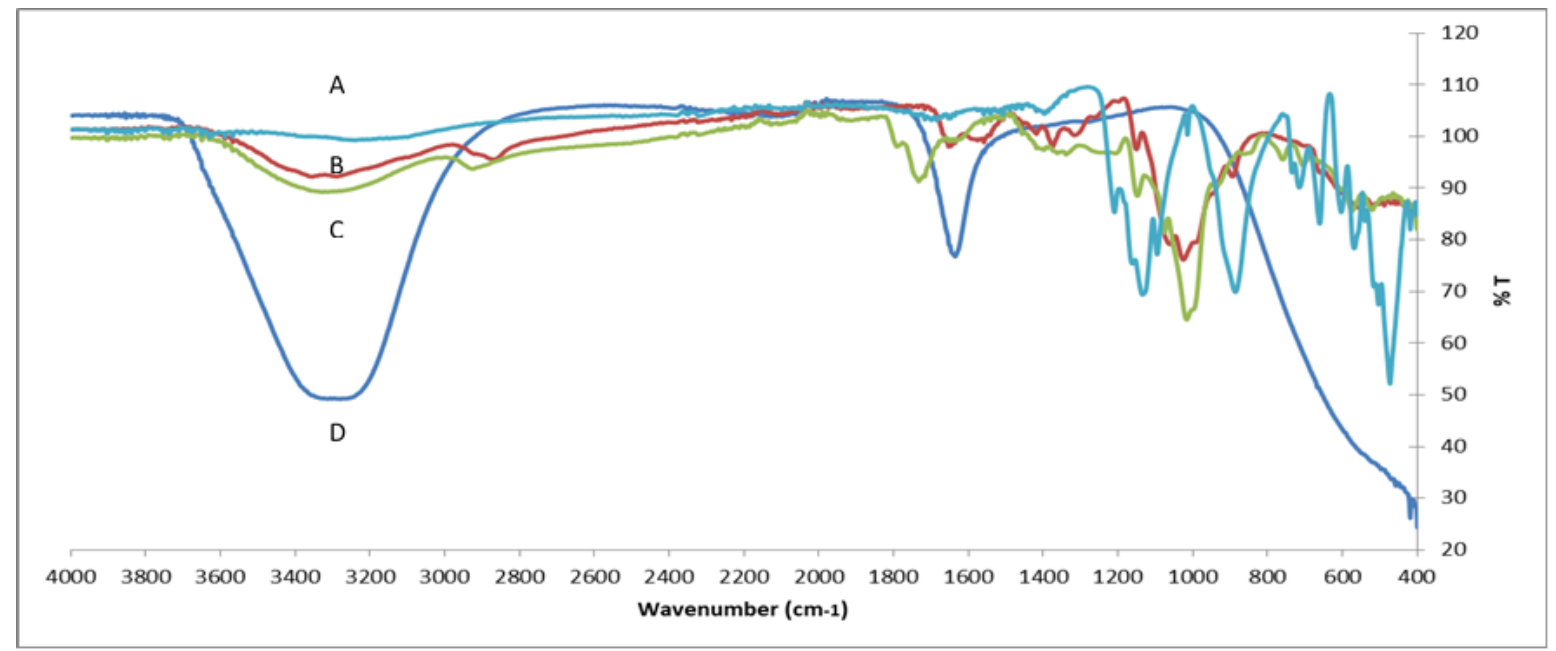

Figure 5. FTIR spectrum of; A) TPP; B) CS; C) Garcinia atroviridis water extract; and D) Garcinia atroviridis loaded CS nanoparticle (CS-TPP 1.5/4:1) 
phenol, and carboxylic acids (Anand et al., 2014). While the band at $2825.72 \mathrm{~cm}^{-1}$ represents the $\mathrm{C}-\mathrm{H}$ stretch of C $\mathrm{O}$ stretching vibration corresponding to aldehydes compound. The band at $1624.06 \mathrm{~cm}^{-1}$ represents the absorption band of $\mathrm{C}=\mathrm{O}$ from the amide group. 1332.881 $\mathrm{cm}^{-1}$ shows the stretching vibration of $\mathrm{C}-\mathrm{O}$ from $\mathrm{CH}-\mathrm{OH}$. The absorption band between 1097 and $1013 \mathrm{~cm}^{-1}$, represent the absorption bands for the free amino group. The band at $979.839 \mathrm{~cm}^{-1}$ shows the stretching vibration of C-O from $\mathrm{CH}_{2} \mathrm{OH}$ (Zhang et al., 2004; Bangun et al., 2018). FTIR spectrum of $G$. atroviridis extract shows a characteristics peak at $3286.70 \mathrm{~cm}^{-1}$ indicating the $\mathrm{OH}$ group; $2887.44 \mathrm{~cm}^{-1}$ indicating the alkane $\mathrm{C}-\mathrm{H} ; 1757.15$ $\mathrm{cm}^{-1}$ referring to the absorption of $\mathrm{O}-\mathrm{C}=\mathrm{O}$ carbonyl group bound to ester where the keto group are attached to ester; and $1008.77 \mathrm{~cm}^{-1}$ representing the group of carbonyl single bond stretch (Choppa et al., 2015). For the CS: TPP nanoparticles, the FTIR spectrum showed an intense and broad absorption band at $3309.85 \mathrm{~cm}^{-1}$ that might be due to the stretching vibration of $\mathrm{O}-\mathrm{H}$. The amount of water present in the CS nanoparticle indicates their hydrophilic properties, which was confirmed through the $\mathrm{O}-\mathrm{H}$ bonding observed in the FTIR spectra between 3200 and $3550 \mathrm{~cm}^{-1}$. The absorption band at $1610 \mathrm{~cm}^{-1}$ was characterized by the bending vibration of N-H in CS-TPP nanoparticles after the addition of TPP. Regarding the $\mathrm{C}-\mathrm{O}$ stretching, the absorption bands of $1624.06 \mathrm{~cm}^{-1}$ in pure CS have shifted to $1610 \mathrm{~cm}^{-1}$ indicating the interaction between CS-TPP nanoparticles (Anand et al., 2018).

\section{Conclusion}

The physicochemical properties of CS nanoparticles can be altered by varying the concentration of chitosan and the volume ratio of chitosan to TPP. Chitosan at a concentration of $1.5 \mathrm{mg} / \mathrm{mL}$ and the volume ratio of $4: 1$ of CS: TPP gave the most optimum nanoparticle suspension with the highest EE. All nanoparticle formulations were found to be non-Fickian diffusion and the HCA release were followed either Higuchi or Korsmeyer Peppas model for the release of the extracts.

\section{Acknowledgements}

The authors gratefully acknowledge the HICOE UTM Grant (R.J130000.7846.4J264) and Zamalah Scholarship for supporting this work.

\section{References}

Anand, M., Kalaivani, M., Maruthupandy, M., Kumaraguru. and Suresh, S. (2014). Extraction and characterization of chitosan from marine crab and squilla collected from the Gulf of Mannar Region,
South India. Journal Chitin Chitosan Science, 2, 280 $-287$.

Anand, M., Sathyapriya, P., Maruthupandy, M. and Hameedha, B. (2018). Synthesis of chitosan nanoparticles by TPP and their potential mosquito larvicidal application. Frontiers in Laboratory Medicine, 2(2), 72-78. https://doi.org/10.1016/ j.flm.2018.07.003

Bangun, H., Stevan, T. and Anayanti, A. (2018). Preparation and evaluation of chitosantripolyphosphate nanoparticles suspension as an antibacterial agent. Journal of Applied Pharmaceutical Science, 8(12), 147-156. https:// doi.org/10.7324/JAPS.2018.81217

Bhumkar, D.R. and Pokharkar, V.B. (2006). Studies on Effect of $\mathrm{pH}$ on .Cross-linking of Chitosan with Sodium Tripolyphosphate: A Technical Note. An Official Journal of the American Association of Pharmaceutical Scientists, 7(2), 138-143. https:// dx.doi.org/10.1208\%2Fpt070250

Braswell, A. and Atlanta, Ga. (1999). Method for controlling weight with Hypericum perforatum and Garcinia cambogia. U.S. Patent No. 5,911,992. Washington, DC: U.S. Patent and Trademark Office.

Ezhilarasi, P., Muthukumar, S. and Anandharamakrishnan, C. (2016). Solid Lipid Nanoparticle Enhances Bioavailability of Hydroxycitric Acid Compared to a Microparticle Delivery System. RSC Advances, 6, 53784-53793. https://doi.org/10.1039/C6RA04312G

Fabio, G. and Marta, M. (2003). Monitoring of Apoptosis of HL60 cells by Fourier-Transform Infrared Spectroscopy. Journal of Biochemical Society, 369(2), 239-248. https://doi.org/10.1042/ bj20021021

Fathi, M., Jaleh, V., Mohebbat, M. and Fakhri, S. (2013). Hesperetin-Loaded Solid Lipid Nanoparticles and Nanostructured Lipid Carriers for Food Fortification: Preparation, Characterization, and Modeling. Food and Bioprocess Technology, 6(6), 1464-75. https:// doi.org/10.1007/s11947-012-0845-2

Gokaraju, R., Rama, R., Ventaka, S. and Sridhar, P. (2005). Process for preparing highly water soluble double salts of hydroxycitric acid particularly alkali and alkaline earth metal double salts. U.S. Patent No. US 6,875,891 B2. Washington, DC: U.S. Patent and Trademark Office.

Hamidi, M., Azadi, A. and Rafiei, P. (2008). Hydrogel nanoparticles in drug delivery. Advanced Drug Delivery Reviews, 60(15), 1638-1649. https:// doi.org/10.1016/j.addr.2008.08.002

Jayaprakasha, K.G. and Sakariah, K.K. (2000). 
Determination of hydroxycitric acid in commercial samples of garcinia cambogia extract by liquid chromatography with ultraviolet deection. Journal of Liquid Chromatography and Related Technologies, 23(6), 915-923. https://doi.org/10.1081/JLC100101498

Jayaprakasha, K.G., Jena, S.B. and Sakariah, K.K (2003). Improved Liquid Chromatographic Method for Determination of Organic Acids in Leaves, Pulp, Fruits, Rinds of Garcinia. Journal of $A O A C$ International, 86(5), 1063 - 1068. https:// doi.org/10.1093/jaoac/86.5.1063

Jena, B., Jayaprakasha, K. and Kunnumpurath, K. (2002). Organic Acids from leaves, Fruits, and Rinds of Garcinia cowa. Journal of Agricultural and Food Chemistry, 50, 3431-3434. https://doi.org/10.1021/ jf011627j

Jonassen, H., Anna, L. and Marianne, H. (2012). Stability of Chitosan Nanoparticle Cross-Linked with Tripolyphosphate. Biomacromolecules, 13, 3747- 3757. https://doi.org/10.1021/bm301207a

Kaszuba, M., Corbett, J. and Watson, A. (2010). Highconcentration zeta potential measurements using light-scattering techniques. Philosophical Transactions of the Royal Society A, 368(1927), 4439-4451. https://doi.org/10.1098/rsta.2010.0175

Kulakovskaya, T.V., Vladimir, M. and Igor, S. (2012). Inorganic polyphosphate in industry, agriculture and medicine. Modern state and outlook. Process Biochemistry, 47(1), 1-10. https://doi.org/10.1016/ j.procbio.2011.10.028

Ling, Y., Noraziah, M., Atif, S. and Haliza, K. (2012). Antifungal Activity of Chitosan Nanoparticles and Correlation with Their Physical properties. International Journal of Biomaterials, 2012, 632698. https://doi.org/10.1155/2012/632698

Lokhandwala, H., Ashwini, D. and Shirish, D. (2013). Kinetics Modelling and dissolution Profiles comparison: an Overview. International Journal of Pharma and Bio Sciences, 4(1), 728-737.

Masaruddin, M.J., Suzanne, M., Evison, B.J., Philips, D.R. and Pigram, P. (2015). Factors determining the stability, size distribution, and cellular accumulation of small, monodisperse chitosan nanoparticles as candidate vectors for anticancer drug delivery: application to the passive encapsulation of [14C]doxorubicin. Nanotechnology, Science and Application, 8, 67-80. https://doi.org/10.2147/ nsa.s91785

Mislick, K.A. and Baldesschwieler, J.D. (1996). Evidence for the Role of Proteoglycans in cationmediated gene transfer. Proceeding of the National
Academy of Sciences of the United States of America, 93(22), 12349-12354. https:// doi.org/10.1073/pnas.93.22.12349

Moffet, S.A., Ashok, K.B., Bhagavathula, R. and Karanam, B. (1997). Hydroxycitric acid concentrate and food products prepared thereform. U.S. Patent No. 5,656,314. Washington, DC: U.S. Patent and Trademark Office.

Mohammed, A., Jaweria, T., Kishor, M. and Ellen, K. (2017). An Overview of Chitosan Nanoparticles and Its Application in Non-Parenteral Drug Delivery. Pharmaceutics, 9(4), 53. https://doi.org/10.3390/ pharmaceutics 9040053

Nagpal. K., Singh, S. and Mishra, D. (2013). Optimization of brain targeted chitosan nanoparticles of Rivastigmine for improved efficacy and safety. International Journal of Biological Macromolecules, 59, 72-83. https://doi.org/10.1016/ j.ijbiomac.2013.04.024

Nemrawi, A., Alsharif, S. and Dave, R. (2018). Preparation of Chitosan-TPP Nanoparticles: The Influence Of Chitosan Polymeric Properties And Formulation Variables. International Journal of Applied Pharmaceutics, 10(5), 60-65. https:// doi.org/10.22159/ijap.2018v10i5.26375

Powers, K., Scott, B.C., Vijay, B., Scott, C., Wasdo, B. and Stephen, M. (2006). Research Strategies for Safety Evaluation of Nanomaterials. Part VI. Characterization of Nanoscale Particles for Toxicological Evaluation. Toxicological Science, 90 (2), 296-303. https://doi.org/10.1093/toxsci/kfj099

Purwantiningsih, S., Laksmi, A. and Lidiniyah. (2015) Optimization of Ketoprofen-loaded chitosan nanoparticles ultrasonication process. Procedia Chemistry, 16, 673-680. https://doi.org/10.1016/ j.proche.2015.12.007

Quan, G., Tao, W., Colette, C. and Paul, M. (2005). Modulation of Surface Charge, Particle Size and Morphological Properties of Chitosan-TPP Nanoparticles Intended for Gene Delivery, Colloids and Surfaces B. Biointerfaces, 44(2-3), 65-73. https://doi.org/10.1016/j.colsurfb.2005.06.001

Rampino, A., Masssimiliano, B. and Paolo, B. (2013). Chitosan nanoparticles: Preparation, size evolution and stability. International Journal of Pharmaceutics, 455(1-2), 219-228. http:// dx.doi.org/10.1016/j.ijpharm.2013.07.034

Roongpisuthipong, C., Kantawan, R. and Roongpisuthipong, W. (2007). Reduction of adipose tissue and body weight: effect of water soluble calcium hydroxycitrate in Garcinia atroviridis on the short term treatment of obese wome in Thailand. 
Asia Pacific Clinical Nutrition, 16(1), 25-29. https://

doi.org/10.6133/APJCN.2007.16.1.04.

Sawtarie, N., Yuhang, C. and Yakov L. (2017). Preparation of Chitosan/Tripolyphosphate Nanoparticles with Highly Tunable Size and Low Polydispersity. Colloids and Surfaces B. Biointerfaces, 157, 110-117. https://doi.org/10.1016/ j.colsurfb.2017.05.055

Semwal, R.B., Deepak, K.S., Ilze, V. and Alvaro, V. (2015). A comprehensive scientific overview of Garcinia cambogia. Fitoterapia, 102, 134-148. https://doi.org/10.1016/j.fitote.2015.02.012

Suvakanta, D., Padala, N., Lilakanta, N. and Prasanta, C. (2010). Kinetics modeling on drug release from controlled drug delivery systems. Acta Poloniae Pharmaceutica-Drug Research, 67(3), 217-223.

Verhoeckx, K., Cotter, P., Lopez-Exposito, I., Kleiveland, C., Lea, T., Mackie, A., Requena, T., Swiatecka, D. and Wichers, H. (Eds.). (2015).The Impact of Food Bioactives on Health: In Vitro and Ex Vivo Models. Switzerland: Springer, Cham. https://doi.org/10.1007/978-3-319-16104-4

Vinagradov, S., Bronich, T. and Kabanov, A. (2002). Nanosized cationic hydrogels for drug delivery: Preparation, Properties and Interactions with cells. Advances Drug Delivery Rev, 54, 223-233. https:// doi.org/10.1016/s0169-409x(01)00245-9

Wang, J., Zeng, Z., Xiao, R., Xie, T., Zhou, G., Zhan, X. and Wang, S. (2011). Recent advances of chitosan nanoparticle as drug carriers. International Journal of Nanomedicine, 6, 765-774. https:// doi.org/10.2147/ijn.s17296.

Xianyi, S., Jie, G., Yanzuo, C. and Xiaoling, F. (2012). Effect of phospholipid composition on pharmacokinetics and biodistribution of epirubicin liposomes. Journal of Liposome Research, 22(1), 8088. https://doi.org/10.3109/08982104.2011.627513.

Zhang, H., Megan, O., Christine, A. and Eugenia, K. (2004). Monodisperse chitosan nanoparticles for mucosal drug delivery. Biomacromolecules, 5, 24612468. https://doi.org/10.1021/bm0496211. 